\title{
Caries en la tercera edad en una universidad de la ciudad de Puebla 2017-2018.
}

\section{Carries in the third age in a university of the City of Puebla 2017-2018.}

\author{
María Margarita Quiroz Hernández,* Eric Contreras Sánchez,* Yadira Thereza Pacheco Paredes ${ }^{\ddagger}$
}

\section{RESUMEN}

La caries es una infección que afecta a cualquier persona sin importar edad, género o clase social. Es importante enfocar la atención odontológica a pacientes de tercera edad ya que tienden a un crecimiento anual de 3.8\% de la población mexicana. Actualmente, se han realizado campañas de salud bucal para esta población; a pesar de los múltiples esfuerzos realizados para la prevención y erradicación de dicha enfermedad existe un descuido en los tratamientos para pacientes de la tercera edad, lo cual puede favorecer la pérdida de dientes. El objetivo del estudio fue determinar la prevalencia y severidad de caries en adultos mayores en la clínica de odontología de la Universidad Popular Autónoma del Estado de Puebla (UPAEP). Se realizó un estudio descriptivo, retrospectivo; se revisaron 345 expedientes de pacientes de tercera edad del 2017 al 2018, que asistieron por primera vez a consulta. Se obtiene la prevalencia y severidad a través del índice CPOD (cariados, perdidos y obturados). La prevalencia de dientes cariados fue $79 \%$; se obtuvo un CPOD de 18.63 (alto riesgo). Se concluye el implementar medidas preventivas que impacten en mayor dimensión a la caries, para su disminución o erradicación en pacientes geriátricos.

Palabras clave: Caries, tercera, edad, cariados, perdidos, obturados.

\section{ABSTRACT}

Caries is an infection that affects anyone regardless of their age, gender or social class. It is, however, important to focus dental care on elderly patients as that part of the Mexican population is growing at 3.8\% annually. Currently, oral health campaigns have been carried out for this population, but despite the multiple efforts undertaken for prevention and eradication of the disease, treatment for elderly patients is often overlooked. The consequence of this lack of treatment can be the loss of teeth. The objective of this study is to determine the prevalence and severity of caries in elderly patients at the dental clinic of the Universidad Popular Autónoma del Estado de Puebla (UPAEP). A descriptive, retrospective study was carried out where the records of 345 elderly, first-time patients were reviewed for the 2017-2018 period. The prevalence and severity of caries was determined using the DMFT index (decay, missing, filled). The prevalence of carious teeth was $79 \%$ with a DMFT of 18.63 indicating high risk. We conclude that preventive measures should be implemented to impact and decrease caries in geriatric patients.

Keywords: Caries, elderly, decay, missing, filled.

\section{INTRODUCCIÓN}

$\mathrm{L}$ a caries es la enfermedad con mayor prevalencia en el mundo que afecta a niños, adultos y a personas de la tercera edad; es clasificada como una de las 10 enfermedades crónicas a nivel mundial, ${ }^{1}$ no tiene predilección de género, afecta a cualquier rango de edad y es considerada

\footnotetext{
* Estudiante de la Facultad de Odontología.

₹ Profesora de la Facultad de Odontología.

Universidad Popular Autónoma del Estado de Puebla (UPAEP).
}

Recibido: 06 Enero 2020.

Aceptado para publicación: 12 Enero 2020. una enfermedad transmisible, irreversible y multifactorial. Tiene interacción con cuatro factores: el huésped, la microflora, el sustrato y el tiempo; ${ }^{2,3}$ agregando múltiples factores fisiológicos, conductuales y ambientales, actuando de manera sinérgica, compleja y dinámica. ${ }^{4,5}$

Actualmente, existe un incremento importante de adultos mayores y es de suma importancia prestar atención a este grupo de edad, dado el último censo del 2010 en México, que arroja que hay 10 millones de adultos mayores de 60 años, correspondiente a $9 \%$ total de la población con un crecimiento anual de $3.8 \%$, para el 2050 se estima que el adulto mayor conformará cerca de $28 \%$ de la población, implicando un rápido envejecimiento de la población. ${ }^{6,7}$ Este aumento de la variable del adulto mayor ha ocasionado que en los últimos años sea una de las prioridades políticas 
sociales y de salud; sin embargo, son pocos los programas que en la actualidad se tienen para este sector de la población, por consiguiente es fundamental la creación de un programa de salud bucal y general propia del adulto mayor. ${ }^{6}$

A pesar de los servicios gratuitos y campañas de salud bucal existe la necesidad de asistir a servicios particulares de bajo costo como son las universidades. El estudio se realizó en la clínica de odontología de la Universidad Popular Autónoma del Estado de Puebla (UPAEP) con pacientes de la tercera edad.

El adulto mayor, como característica de su edad y del proceso de envejecimiento, asume determinados comportamientos psicosociales y condicionan la actitud en la consulta odontológica y por ende la percepción sobre el servicio. Se reconoce que por naturaleza los adultos mayores se hacen más vulnerables a presentar problemas en la salud y a su vez pueden mostrar una mayor probabilidad de tener más limitantes para acceder a la resolución y control de la enfermedad. ${ }^{8}$

A pesar de las campañas preventivas realizadas en la disminución y eliminación de la caries, es evidente la pérdida de dientes y la falta de rehabilitación pertinente, lo que afecta la salud oral. Esto se ve influenciado por la situación de vulnerabilidad a los cambios naturales del envejecimiento físico como: disminución del volumen y peso corporal, rigidez muscular, fragilidad ósea y problemas de multimorbilidad afectando directamente a la desnutrición e higiene personal, por lo cual también presentan algún grado de deterioro cognitivo, por lo tanto ocasiona el aumento de limitantes de desplazamiento de un lugar a otro e incrementa el riesgo de contraer un accidente por factores externos. ${ }^{9,10}$ Por otro lado, se encuentran los factores sociales y psicológicos que van apareciendo cuando el individuo comienza a perder sus funciones ya antes mencionadas y por ello aparece el aislamiento, lo cual lo restringe de sus actividades e impide su participación activa. ${ }^{11}$ Un gran porcentaje de adultos mayores padecen de depresión y ansiedad por factores como las enfermedades adquiridas, el entorno en el que viven, estado civil y la soledad, incluyendo la falta de trabajo. ${ }^{12}$ Otras limitantes influyentes en el adulto mayor que repercuten directamente en el descuido personal es el abandono y la falta de atención familiar; ${ }^{13,14}$ debido al transcurso del tiempo, los adultos mayores son considerados una carga extra para los familiares debido al padecimiento de múltiples enfermedades e incremento en el cuidado hacia el adulto mayor. ${ }^{14}$ Las personas de la tercera edad se tornan dependientes de sus familiares, impactando directamente en el descuido personal y bucodental en este rango de edad, esto influye en la captación de la enfermedad y aceptación del tratamiento, así como la buena comunicación con los prestadores de salud. ${ }^{14}$ Es importante concientizar a los adultos mayores y a los familiares para tener un mejor cuidado en su cavidad oral, debido a las complicaciones que éstas pueden provocarles en la salud sistémica. ${ }^{15,16}$

El objetivo del trabajo fue determinar la prevalencia y severidad de los órganos dentarios más susceptibles a caries en adultos mayores, en la clínica de odontología UPAEP.

\section{MATERIAL Y MÉTODOS}

Se realizó un estudio descriptivo, transversal, retrospectivo y observacional con 345 expedientes del archivo de la Clínica Odontológica, en pacientes de 60 años y más del 2017 al 2018, que asistieron por primera vez.

Se solicitó autorización ante las instancias pertinentes para el uso y recolección de los expedientes clínicos, solicitando el permiso y consentimiento para el uso de esta información, por lo cual se asignaron días específicos y se diseñó la hoja de recolección de datos en Excel. En cada revisión de expedientes, se procedió al registro de los hallazgos de la historia clínica en la hoja de recolección de datos. Se elaboraron cuadros de contingencia permitiendo analizar la prevalencia y severidad existente de caries en el adulto mayor.

\section{RESULTADOS}

Se revisaron 345 adultos mayores de 60 años y más que asistieron en el año 2017 y 2018 a la clínica odontológi-

\begin{tabular}{|c|c|c|c|c|c|c|}
\hline Género & 2017 & $\%$ & 2018 & $\%$ & $2017-2018$ & $\%$ \\
\hline Femenino & 119 & 67 & 102 & 61 & 221 & 64 \\
\hline Masculino & 59 & 33 & 65 & 39 & 124 & 36 \\
\hline Total & 178 & 100 & 167 & 100 & 345 & 100 \\
\hline
\end{tabular}


ca, $64 \%$ de la población fueron mujeres y $36 \%$ hombres (Tabla 1).

La prevalencia de dientes cariados en pacientes adultos mayores fue de 79\%; en el periodo 2017 fue de 75\% y en el año 2018 fue de $83 \%$.

En los órganos dentarios perdidos se muestra una prevalencia de $85 \%$; en el periodo 2017 la prevalencia fue de $82 \%$ y en el año 2018 fue de $88 \%$.

La prevalencia de órganos dentales obturados fue de 62\%; en el 2017 fue de 59 y 66\% en el 2018 (Tabla 2).

En los resultados se encontró que de los 345 pacientes, el diente con mayor afectación por caries fue el 34 con un $29.85 \%$ de la población, y el diente que presentó mayor porcentaje de pérdida fue el 36 con un $54.78 \%$ de los casos (Figura 1).

El índice CPO se distribuye por edades de ambos sexos del año 2017 y 2018 con un global de 18.63 (Tabla 3 y 4). Se aprecian índices de severidad de alto riesgo en

\begin{tabular}{cccc}
\multicolumn{4}{c}{ Tabla 2: Distribución de prevalencia. } \\
\hline & $2017(\%)$ & $2018(\%)$ & 2017-2018 (\%) \\
\hline C & 75 & 83 & 79 \\
P & 82 & 88 & 85 \\
O & 59 & 66 & 62 \\
\hline
\end{tabular}

los pacientes de 60 a 64 años de edad con un índice de 6.3 y en pacientes de 80 a 100 años con un bajo riesgo (Tabla 4).

\section{DISCUSIÓN}

La prevalencia de caries en el presente estudio realizado en la clínica de odontología de la UPAEP fue alta en adultos mayores, posiblemente asociado a factores relacionados al envejecimiento tales como: problemas psicológicos (depresión y ansiedad), multimorbilidad, rigidez corporal, fragilidad ósea, deterioro cognitivo, la falta de atención y abandono de familiares hacia estos; ocasionando el descuido personal y dental junto con otros factores como el nivel socioeconómico y educativo. ${ }^{10} \mathrm{Al}$ comparar los resultados obtenidos en Puebla, 79\% de adultos mayores afectados por caries, con el SIVEPAB (Sistema de Vigilancia Epidemiológica de Patologías Bucales) $98 \%$ de pacientes de la tercera edad con problema de caries a nivel nacional, ${ }^{17}$ se deduce que este sector vulnerable requiere mayor atención para el control de los padecimientos bucodentales como es la caries.

$\mathrm{Al}$ estudiar el riesgo a caries en Puebla, arroja el 18.63. En un estudio realizado en el estado de Hidalgo obtuvieron un índice de $19.7,{ }^{18}$ considerándose en ambos casos un riesgo alto. Por lo tanto, se deben implementar estrategias educativas, preventivas e interceptivas en las poblaciones más vulnerables como el paciente geriátrico

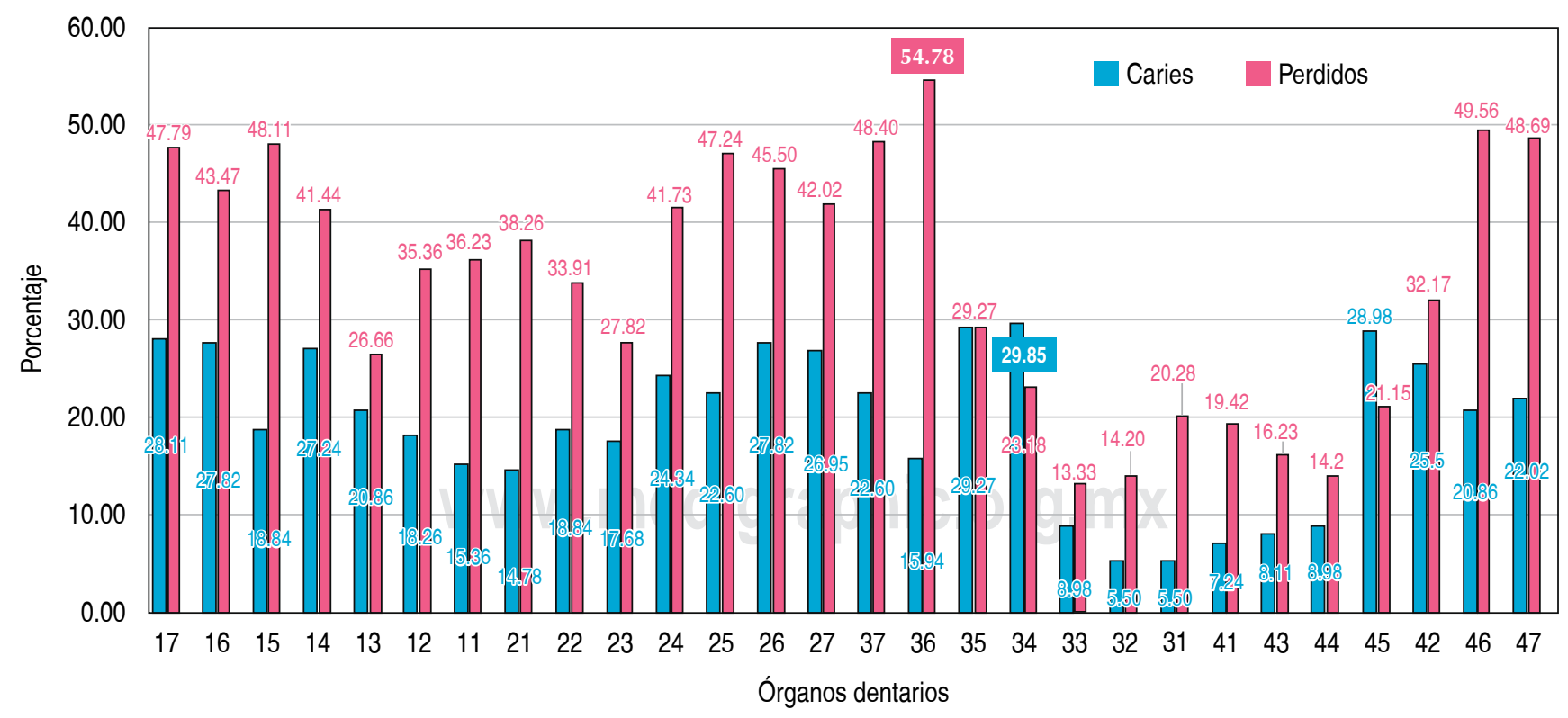

Figura 1: Órganos dentarios más afectados por caries y perdidos. 
Tabla 3: Distribución de índice CPO.

\begin{tabular}{cccc} 
& 2017 & 2018 & $2017-2018$ \\
\hline CPO & 18.12 & 19.16 & 18.63 \\
\hline
\end{tabular}

Tabla 4: Distribución por edades de ambos sexos 2017-2018.

\begin{tabular}{cc} 
Edad & CPO \\
\hline $60-64$ & 6.3 \\
$65-69$ & 3.99 \\
$70-74$ & 4.52 \\
$75-79$ & 2.17 \\
$80-84$ & 1.08 \\
$85-100$ & 0.57 \\
Total & 18.63 \\
\hline
\end{tabular}

ofreciendo una atención transdisciplinaria, mejorando la calidad de vida. Se pretende disminuir o erradicar la caries en estos pacientes para evitar la pérdida de órganos dentarios y al estar en una etapa susceptible por el envejecimiento puede favorecer los procesos de desnutrición, provocando una función masticatoria deficiente. ${ }^{4}$ Esta última afecta la digestión y la absorción de los nutrientes necesarios para el mantenimiento, crecimiento y desarrollo de las funciones. ${ }^{10}$ La pérdida de dientes favorece en esta población diversas patologías bucodentales, como la pérdida de dimensión vertical, que puede derivar a problemas temporomandibulares, queilitis angular y maloclusión dental cuando no es rehabilitado. ${ }^{3,13}$

La salud oral en México ha progresado en pacientes de la tercera edad, sin embargo, se deben realizar más estudios epidemiológicos en esta población, debido al actual ascenso de la población adulta mayor de 3.8\% y un futuro incremento total de $28 \%{ }^{6,7}$

\section{CONCLUSIONES}

Es necesario brindar atención transdisciplinaria al paciente geriátrico ya que el cuidado bucal es complejo, esto implica establecer adecuaciones en su manejo por tratarse de pacientes con problemas sistémicos, sociales, físicos y mentales, diferentes a la población joven. Una de las enfermedades más frecuentes en los adultos mayores es la caries, al igual que las enfermedades periodontales, siendo relevante concientizar a la edad adulta a implementar hábitos higiénicos adecuados para el mejor mantenimiento de la cavidad oral. Existen múltiples barreras motoras en el adulto mayor que no permiten el adecuado cepillado dental e interdental, que requieren la ayuda de familiares, amigos o conocidos, pero no todas las personas de la tercera edad cuentan con el apoyo social, por ello tienen un sensible deterioro oral. Por esta razón, se deben realizar más estudios epidemiológicos a este grupo de riesgo para evaluar el estado de su salud oral en cada paciente y así reducir el riesgo a caries, periodontitis y la pérdida dental.

Es fundamental concientizar a las instituciones públicas y privadas a efectuar un mayor esfuerzo preventivo y educativo para potencializar aquellas estrategias epidemiológicas en los servicios odontológicos, siendo prioritario establecer programas multidisciplinarios e interdisciplinarios alcanzables para erradicar aquellas barreras, las cuales impiden al paciente cumplir su tratamiento y mantenimiento.

\section{BIBLIOGRAFÍA}

1. Chu $\mathrm{CH}, \mathrm{Ng} \mathrm{A}, \mathrm{Chau} \mathrm{AM}$, Lo EC. Oral health status of elderly chinese with dementia in Hong Kong. Oral health \& prev dent [internet]. 2015 Feb 24 [cited 2019 May 22]; 13 (1): 51-7. Available from: https://ohpd.quintessenz.de/index.php?doc =abstract\&abstract $\mathrm{ID}=32343 /$.

2. Rodríguez T, Hernández DI, Lara A. Bioquímica de la caries. Rev Mex de Estoma [internet]. 2018 Jun 30 [cited 2019 Apr 13]; 5 (1): 6-7. Available from: https://www.remexesto.com/index.php/ remexesto/article/view/180/362.

3. Hernandez L, Marimón M, Linares E, Gonzalez E. Salud oral y hábitos dietéticos en los adultos mayores no institucionalizados. Rev Cubana Aliment Nutr [Internet]. 2017 Feb 23 [cited 2019 Jun 13]; 27 (1): 29-48. Available from: https://www.medigraphic.com/ pdfs/revcubalnut/can-2017/can171d.pdf.

4. González AM, González BA, González E. Salud dental: relación entre la caries dental y el consumo de alimentos. Nutr Hosp [Internet]. 2013 Jul [cited 2019 Apr 23]; 28 (4): 64-71. Available from: http://scielo.isciii.es/scielo.php?script=sci_arttext\&pid $=$ S0212-16112013001000008.

5. Maykanathan D, Kaur S. Socio-demographics, Oral Health Behaviour, and Physical Activity: Factors in Caries Experience among 19-59 Years Old Adults in a Malaysian. Makara Jour Health Res [internet]. 2015 Aug [cited 2019 May 24]; 19 (2): 55-60. Available from: http://journal.ui.ac.id/index.php/health/article/view/4854.

6. Rodríguez BM, Cruz P, Méndez J. Necesidades de tratamiento dental y/o periodontal en adultos mayores en una población mexiquense. Rev Hor [internet]. 2014 Aug 28 [cited 2019 Aug 3]; 49-59. Available from: http://web.uaemex.mx/revistahorizontes/ docs/revistas/Vol6/4_NECESIDADES.pdf.

7. Hernández S, de la Fuente YM. Análisis competencial de la atención a la dependencia en los adultos mayores desde el ámbito institucional de México. Gest Polít Púb [Internet]. 2015 Mar 2 [cited 2019 Aug 7]; 25 (1): 299-305. Available from: http://www.scielo. org.mx/pdf/gpp/v25n1/v25n1a8.pdf. 
8. Agudelo AA, Alzate S, Lopez F, Lopez C, Espinosa E. Barreras y facilitadores de acceso a los servicios de salud bucal para la población adulta mayor atendida en la red pública hospitalaria de Medellín, Colombia. Rev Gerenc Polít Salud [Internet]. 2014 Abr 1 [cited 2019 Aug 7]; 13 (27): 181-199. Available from: http://www. scielo.org.co/pdf/rgps/v13n27/v13n27a11.pdf.

9. Quino AC, Chacon MJ. Capacidad funcional relacionada con actividad física del adulto mayor en Tunja, Colombias. Hor san [Internet].2018 Ene [cited 2019 Jun 25]; 17 (1): 59-68. Available from: http://www.scielo.org.mx/scielo.php?script=sci abstract\&pid=S2007-74592018000100059\&lng=es\&nrm =iso.

10. Arriola E, Carnero C, Freire A, Lopez R, López JA, Manzano S, Olazarán J. Deterioro cognitivo leve en el adulto mayor. Sdad Esp de Geria y Gero [Internet]. 2017 [cited 2019 Aug 10]; 13 (1) 6-8. Available from: https://www.segg.es/media/descargas/Consenso $\% 20$ deteriorocognitivoleve.pdf.

11. Calderón D. Epidemiología de la depresión en el adulto mayor. Rev Med Herediana [Internet]. 2018 Jul [cited 2019 Sep 1]; 29 (3): 182-187. Available from: http://www.scielo.org.pe/scielo. php?script=sci_arttext\&pid=S1018-130X2018000300009.

12. Roberts A, Ogunwole S, Blakeslee L. The Population 65 Years and Older in the United States: Unit Stat Cens Bureau [Internet]. 2018 Oct [cited 2019 Aug 31]; 38. Available from: https://www.census. gov/content/dam/Census/library/publications/2018/acs/ACS-38.pdf.

13. Cárdenas A, Velazquez LB, Falcón JA, García IE, Montes RE. Autopercepción de la salud oral en adultos mayores de la Ciudad de México. Rev Med Inst Mex Seguro Soc [Internet]. 2018 Nov [cited 2019 Sep 25]; 1(54): S54-S58. Available from: https://www. medigraphic.com/pdfs/imss/im-2018/ims181h.pdf.
14. Ashwin M, Kirubagaran S, Dhanraj M. Oral hygiene measures among elderly people in the destitute homes in Chennai - A survey. Drug Invent Today [Internet]. 2018 Oct 17 [cited 2019 Sep 30]; 11(2): 278-281. Available from: http://jprsolutions.info/files/finalfile-5c56798ae07141.93680200.pdf.

15. Habib G, Lancellotti P, Antunes MJ et al. Guía ESC 2015 sobre el tratamiento de la endocarditis infecciosa. Rev Esp Cardiol [Internet]. 2016 [cited 2019 Oct 3]; 1-49. Available from: https://www. revespcardiol.org/es-pdf-S030089321500651X.

16. Ceballos $\mathrm{H}$, Rodríguez LF, Bobadilla A. Profilaxis antimicrobiana previa a procedimientos dentales. Acta Pediatr Mex [Internet]. 2017 Jul 28 [cited 2019 Oct 25]; 38 (5): 337-350.Available from: https:// www.medigraphic.com/pdfs/actpedmex/apm-2017/apm175g.pdf.

17. Lomeli G, Rodriguez KG. Patologías Bucales Resultados del Sistema de Vigilancia Epidemiológica de SIVEPAB 2017. Cent Nac de Prog Prevent y Contr de Enf [Internet]. 2017 [cited 2019 Nov 14]; 1 (1): 89-92. Available from: https://www.gob.mx/cms/uploads/ attachment/file/459279/SIVEPAB_2017.pdf.

18. Cortés X, Hernandez R, Olga A. Perfil de salud bucodental en un grupo de adultos mayores del estado de Hidalgo. Rev ADM [Internet]. 2014 Feb [cited 2019 Nov 13]; 71 (2): 77-82. Available from: https://www.medigraphic.com/pdfs/adm/od-2014/od142f. pdf.

Correspondencia:

Eric Contreras Sánchez

E-mail: eric.contreras@upaep.edu.mx 